\title{
Diagnóstico del aprendizaje táctico en la selección nacional feme- nina de balonmano, categoría juvenil
}

Lenigan Jaime Banegas ${ }^{1}$

Xabel Secades ${ }^{2}$

\section{RESUMEN}

La presente investigación parte de las insuficiencias que se manifiestan en la inadecuada utilización del diagnóstico como instrumento de medición del aprendizaje de los contenidos tácticos en el balonmano femenino, categoría juvenil de Honduras. Lo cual se orienta el objetivo a elaborar una metodología para contribuir al desarrollo del diagnóstico del aprendizaje táctico en dichas jugadoras. En este sentido se define como objeto de investigación: el proceso de evaluación en las jugadoras del equipo balonmano y como campo de acción: el diagnóstico del aprendizaje táctico. En post de alcanzar su finalidad organizada a partir de las tareas investigativas se utilizaron métodos teóricos y empírico, así como técnicas de investigación, al permitir evidenciar las carencias desde el punto de vista científico que dieron lugar a formular el problema y contribuyeron a constatar no solo los resultados del diagnóstico, sino las valoraciones finales a través del criterio de especialistas. La novedad científica radica en la estructuración de la metodología para el diagnóstico del aprendizaje táctico, desde una perspectiva sistémica, al contextualizar su aplicación en los diferentes momentos de la formación de las jugadoras. Dota al proceso de enseñanza aprendizaje de un conjunto de ejercicios e instrumentos basados en registros de medición, que favorecen el desempeño profesional de los entrenadores para determinar el desarrollo alcanzado por estas en cada etapa de la preparación.

Palabras clave: diagnóstico, aprendizaje, táctico, balonmano, evaluación.

\footnotetext{
${ }^{1}$ Estudiante de Maestría en Actividad Física Entrenamiento y Gestión deportiva, en la Universidad Europea Del Atlántico; Santander España. Profesor de Educación Física con Orientación en la Docencia, Facultad de Humanidades y Arte, Escuela de Ciencias de la Cultura Física, UNAH: lenigan.jaime@unah.edu.hn

${ }^{2}$ Asesor, área de Deportes FUNIBER - Fundación Universitaria Iberoamericana Barcelona (España):tutor29.sn@funiber.org
} 


\section{ABSTRACT}

The present investigation starts from the inadequacies manifested in the use of the diagnosis as an instrument of measurement of the learning of the tactical contents in the feminine handball, category youth of Honduras. This is aimed a methodology to development tactical learning diagnosis in these players. In this sense, the object of investigation is defined as the evaluation process in the players of the handball team and a field of action: the diagnosis of a tactical learning. In order to achieve its purpose organized from the investigative tasks were used theoretical and empirical methods, as well as research techniques, allowing to show the gaps from the scientific point of view that led to formulate the problem and contributed to verify not only The results of the diagnosis, but the final evaluations through the criterion of specialists. The scientific novelty lies in the structuring the methodology for the diagnosis of tactical learning, from a systemic perspective, when contextualizing its application in different moments of the formation of the players. It endows the teaching process with a set of exercises and instruments based on measurement records, which favor the professional performance of the coaches to determine the development achieved by them at each stage of preparation.

Keywords: diagnosis, learning, tactical, handball, evaluation. 


\section{INTRODUCCIÓN}

En la actividad deportiva el proceso de enseñanza-aprendizaje evidencia transformación y perfeccionamiento constante, en correspondencia con los avances científicos y tecnológicos que han incidido en todos los campos del saber, con el fin de mejorar la calidad formativa y competitiva de los atletas. De este modo, se contribuye a las diversas aristas del desarrollo humano, a la vez que se logra su compromiso con la sociedad en su conjunto.

Desde esta perspectiva, el balonmano, como disciplina de equipo, se caracteriza por la lucha entre contrarios. El intercambio se sustenta en los principios de cohesión y comunicación, ambos inciden en el rendimiento y dinamismo al asegurar la armonía distintiva del juego, a partir de los fundamentos tácticos esenciales.

Para conocer cómo marcha el aprendizaje táctico de las jugadoras se necesita evaluar constantemente cómo este se comporta en la actividad deportiva. Es por ello que el diagnóstico pedagógico cobra singular importancia como instrumento de medición para entrenadores y personal técnico del deporte balonmano.

El diagnóstico es un proceso encaminado a la búsqueda de un conocimiento acerca de cómo marchan los acontecimientos y en qué sentido hay que dar inicio o seguir desarrollando la actividad sujeto del mismo, para poder, mediante un amplio y profundo análisis, llegar a una posible explicación de las características, logros, dificultades y causas de los problemas, con un último propósito de proyectar posibles soluciones para eliminar, atenuar o compensar las dificultades; así como usar de forma conveniente potencialidades o fortalezas también encontradas, es decir, constituye un elemento de control eficaz.

A través del diagnóstico del aprendizaje, aplicado de forma sistemática y certera se puede evaluar el comportamiento del componente táctico de las jugadoras de la selección nacional de balonmano femenino de Honduras.

De manera particular, el componente táctico ha sido abordado por disímiles autores: Refoyo (2001), Espar y Gerona (2004), Tordosa (2006), Mesa (2007), Román (2008), Roldán (2009), Martínez (2010), Antón (2010), Lasierra (2012) y otros. Sus estudios aportan: modelos, estrategias, metodologías, sistemas de ejercicios e indicadores; en lo específico, dan cuenta de la necesidad de profundizar en la evaluación de la táctica. 
Sin embargo, sus propuestas se centran en el empleo de medios para la evaluación técnico-táctica y el control del rendimiento deportivo, no lo analizan como vía de medición del aprendizaje. Tal percepción refleja el insuficiente reconocimiento de sus especificidades, al decir además que, en la práctica los test pedagógicos a realizar implican las características de los aspectos técnicos; por consiguiente, priman la repetición y la esquematización. Lo anterior evidencia insatisfacción en las necesidades del entrenamiento de balonmano.

En coincidencia con la perspectiva de esta investigación, se destacan los estudios recientes de Cordovés (2015), los cuales ponderan los aspectos grupales y colectivos, de la preparación táctica y dan cuenta de los sustentos teóricos para su evaluación. No obstante, la naturaleza de su aporte práctico, consistente en una estrategia didáctica, que en toda medida no satisface las necesidades didácticas del contexto. Dicha estrategia centra su atención en la movilización de los recursos para asegurar su desarrollo y su limitación radica en la carencia de medios y procedimientos para establecer los nexos entre el resto de los componentes del proceso.

Las limitantes descritas dificultan la satisfacción de las necesidades propias de un diagnóstico pedagógico de los diferentes momentos referenciales de una categoría competitiva determinada. Con lo anterior coinciden las valoraciones de entrenadores de balonmano del país, expresadas en una entrevista. A la vez, se confirma a través del análisis de documentos metodológicos, la caracterización de los practicantes de la categoría escolar, así como de la observación realizada a la organización del proceso de enseñanza aprendizaje de este deporte.

A partir de la experiencia personal del investigador y a través del diagnóstico fáctico en la investigación se detectaron las siguientes insuficiencias:

- Limitaciones en el empleo adecuado del diagnóstico por los entrenadores, atendiendo a los diferentes momentos de intervención en el proceso

- Insuficientes medios que, a partir de los instrumentos existentes, contemplen las particularidades del equipo de balonmano y los contenidos trascendentes de dicha preparación

- Pobre reconocimiento para elegir y reelaborar ejercicios que posibiliten el diagnóstico del aprendizaje táctico en las jugadoras

- Insuficiente preparación profesional de los entrenadores en cuanto a la aplicación del diagnóstico del aprendizaje táctico 
En relación con todo lo planteado se propone abordar como problema científico: ¿cómo contribuir al diagnóstico del aprendizaje táctico en el equipo de balonmano categoría juvenil sexo femenino de Honduras?

\section{METODOLOGÍA}

La selección nacional de Honduras, cuya función social responde a la formación integral de los deportistas relacionados con los resultados educativos - deportivos. Se encuentra ubicada en la Villa Olímpica. Dicha institución se subordina a los organismos CONDEPA y Comité Olímpico Hondureño.

Asume como objetivo fundamental asegurar la formación integral de los atletas en su tránsito al alto rendimiento en las categorías iníciales de la pirámide deportiva. A partir de su visión directiva, organiza, ejecuta y controla el proceso docente-educativo desde la formación académica y deportiva en las diferentes disciplinas entre las que se encuentra el balonmano.

Tipo de experimento: cuasi experimento de series cronológicas con intervención pedagógica, natural y formativa de control mínimo.

Objeto: Medir la efectividad de la metodología para el desarrollo del diagnóstico del aprendizaje táctico en la selección nacional femenina de balonmano, categoría juvenil.

Objetivo específico: determinar la eficiencia del diagnóstico del aprendizaje táctico en la selección nacional femenina de balonmano, categoría juvenil.

Unidad experimental: equipo de balonmano femenino de la categoría juvenil de Honduras. Las jugadoras promedian una edad de 13.8 años, con un tiempo de práctica del deporte de 3.6 años y 1.2 en el alto rendimiento. Los roles en que se desempeñan las jugadoras en el equipo se distribuyen de la siguiente manera:

\begin{tabular}{|c|c|c|c|c|c|c|c|}
\hline Centrales & Laterales & Extremos & Pívot & Porteras & $\begin{array}{c}\text { 1ra } \\
\text { Línea }\end{array}$ & $\begin{array}{c}\text { 2da } \\
\text { Línea }\end{array}$ & Total \\
\hline 3 & 5 & 3 & 2 & 2 & 8 & 7 & 15 \\
\hline
\end{tabular}


Se seleccionó de manera intencional el equipo de balonmano femenino perteneciente a la categoría juvenil de Honduras, integrado por 15 jugadoras. Forman parte también de la muestra 9 entrenadores y directivos del balonmano que, a partir de sus experiencias, se consideran especialistas competentes en la temática. Todos son licenciados en Educación Física, de ellos tres ostentan la categoría de Máster; alcanzan una experiencia de siete años o más como entrenadores del alto rendimiento, mientras el promedio de edad es de 32.7 años.

A pesar de que a esta edad la voluntad y la atención son más concentradas, las jugadoras realizan movimientos completos, son perseverantes ante una mala ejecución del ejercicio o las acciones, el 15\% de ellas no tienen decisión propia para solucionar varios ejercicios complejos. No obstante se caracterizan por ser alegres, combativas, flexibles, en la práctica se manifiestan con gran hiperactividad. Mantienen buenas relaciones entre ellas y con los entrenadores, participan activamente en las actividades programadas por el centro escolar.

Las condiciones de vida son aceptables, aunque la situación económica no es la más favorable; el clima familiar, en muchas ocasiones no es el más adecuado y la alimentación en pocas ocasiones se corresponde con las necesidades biológicas para estas edades, sobre todo cuando se enfrentan a la realización de actividades físicas intensas.

\section{Resultados de la evaluación de la pertinencia de la metodología a través de la consulta a los especialistas.}

Según Pérez (2001), se entiende como "especialista" a un individuo, capaz de ofrecer con buena competencia, valoraciones conclusivas sobre un determinado problema, hacer pronósticos reales y objetivos sobre efecto, aplicabilidad, viabilidad y relevancia que pueda tener en la práctica la solución propuesta y brindar recomendaciones. Para la aplicación del método se tienen en cuenta los siguientes pasos:

1. Selección de los posibles especialistas, a través de una encuesta para la determinación de su competencia referente al tema

2. Intercambio con los especialistas en función de obtener sus consideraciones en los aspectos esenciales de la metodología, atendiendo a los siguientes indicadores:

a) Funcionalidad de la metodología

b) Explicación de las fases

c) Originalidad, novedad científica y aplicabilidad de la metodología 
3. Procesamiento y valoración cualitativa de la información recopilada

Para seleccionar los especialistas potenciales se determinaron los siguientes criterios a través de la encuesta: poseer experiencia deportiva, ocho años o más de trabajo como entrenador de balonmano en el alto rendimiento o la base, estudios de postgrado 0 cursos especializados relacionados con el entrenamiento de balonmano y experiencia en contextos internacionales.

En la encuesta realizada a los especialistas, los resultados obtenidos al evaluar su competencia referente al tema se consideró pertinente la cifra de 18 de un total de 24 encuestados, la cual representa el $75 \%$.

Relativo a la experiencia laboral, el promedio es de 13.2 años en el desempeño como entrenadores de balonmano. De ellos, 11 en el alto rendimiento, dato que representa el $61.11 \%$ de la totalidad de los especialistas escogidos. El $100 \%$ ha practicado el deporte e interactuado en contextos internacionales.

A los especialistas seleccionados se les aplicó una encuesta dirigida a evaluar la factibilidad de la metodología con su aparato cognitivo y metodológico. En lo específico, centraron su atención en aspectos como: la funcionalidad y la explicación de las fases. Así como, la novedad científica, originalidad y aplicabilidad de la metodología. Las sugerencias y criterios recibidos posibilitaron el constante perfeccionamiento hasta la concreción final.

\section{Criterio de los especialistas encuestados}

Funcionalidad de la metodología: 11 especialistas, que representan el 61.11\%, consideran positiva su correspondencia a la problemática y ofrece tratamiento particular al balonmano de manera muy favorable. En tanto contribuye, desde la estructura y articulación de sus elementos a la preparación táctica del equipo. Destacan la correspondencia del aporte con las exigencias de la práctica del deporte en cuestión. En este aspecto, los siete restantes la consideran favorable mostrando su aceptación. Explicación de las fases: seis especialistas aprecian muy favorable la relación entre las fases y valoran que es de fácil comprensión, a partir de la explicación ofrecida. De igual modo, nueve encuestados la asumen como favorable. Difieren tres especialistas al considerar poco favorable la explicación de las fases. Aunque esta posición no se consideró relevante (por representar solo el 16.66\%), instó al perfeccionamiento de la propuesta en este indicador. 
Novedad científica, originalidad y aplicabilidad de la metodología: 12 de los especialistas que representan el $66.66 \%$ asumen como novedosa y muy original la metodología. Reconocen la calidad de los ejercicios propuestos y los registros de medición, al constituir instrumentos valiosos para desarrollar el diagnóstico del aprendizaje táctico, en manos de los entrenadores. Al mismo tiempo, enfatizan en la arista metodológica como esencia misma de su desempeño y reconocen el aporte de la investigación como un paso importante para nuevos estudios acerca de la táctica. Destacan su aplicabilidad, pues da muestra de la utilidad de la propuesta.

Los resultados de concordancia entre los especialistas se tienen en cuenta a partir del contraste entre los indicadores expuestos. Es evidente el predominio de los valores cualitativos con la categoría de muy favorables, representando el 53.70\%. Los criterios favorables simbolizaron el $40.74 \%$, mientras los poco favorables constituyen el 5.56\%. En consecuencia, es posible inferir que la metodología para diagnosticar el aprendizaje táctico en el equipo de balonmano 13-15 años sexo femenino, desde el punto de vista cognitivo y metodológico es factible de ser implementada.

\section{Resultados de la implementación de la metodología para el diagnóstico del aprendizaje táctico.}

\section{Desarrollo de la fase preparatoria}

A partir de la socialización en la preparación metodológica se familiariza al entrenador con las principales variantes a diagnosticar en el aprendizaje táctico, según su ubicación en el macro ciclo de entrenamiento. De igual modo, se logra la preparación de los agentes involucrados, a través del intercambio.

En estas actividades, se intercambiaron las ideas principales, atendiendo a los intereses pretendidos en la periodización escogida. Los planteamientos dan muestra de una aceptable apropiación del conocimiento, tras la retroalimentación del estudio constante. También se valoraron los aspectos positivos, negativos e interesantes acerca del tema y se asumieron recomendaciones esenciales. Las cuales fueron aceptadas por los entrenadores, en tanto favorecen la prevención de inclemencias en el proceso.

Durante la socialización se determinó y valoró los objetivos que corresponden a la táctica del equipo. Los entrenadores interactuaron con el material metodológico, que incluye los registros de medición y los medios para realizar el diagnóstico en el entrenamiento. Es notoria la proposición de ajustes dirigidos a la comprensión. Por otra 
parte, de un total de cinco registros se transformaron cuatro los que representaron el $80 \%$ del total. Este aspecto revela la participación en la elaboración de herramientas, atendiendo a sus características y necesidades, desde la construcción colectiva de la metodología, así como el intercambio entre los usuarios potenciales y el investigador. La familiarización de las jugadoras del equipo, se realizó a partir de la conversación grupal, comunicándosele la propuesta del cuasi experimento y sus intenciones; al mismo tiempo fueron descritas con profundidad las ventajas del diagnóstico para la calidad del aprendizaje táctico, además de enfatizar la importancia de ahondar en los presupuestos teóricos que lo sustentan. El equipo en su totalidad manifestó su conformidad e interés por participar de forma activa en cada etapa de la experimentación.

El diagnóstico de los conocimientos teóricos acerca de la táctica, se realizó a partir de la aplicación del test que muestra los resultados obtenidos por cada jugadora. La información muestra un nivel alto en este indicador, en correspondencia con la edad deportiva. El resultado promedio del test se enmarca en el rango entre $61 \%$ y $80 \%$, según la escala de evaluación utilizada.

\section{Desarrollo de la fase de ejecución del diagnóstico táctico (aplicación de series cronológicas)}

1ra serie: ubicada en el 2do meso ciclo básico desarrollador en la etapa de preparación general, del periodo preparatorio, micro ciclo 11.

Objetivo de la preparación táctica en ese momento: realizar acciones tácticas ofensivas de creación de espacios con la intervención de dos jugadoras, desde la defensa lograr el cambio de oponente así como el apoyo en el control del adversario.

\section{Resultados del diagnóstico en la 1ra serie}

Consistencia: se percibieron dificultades en la consistencia del aprendizaje, a través de la aplicación de situaciones tácticas ofensivas de 3vs3, atendiendo a las relaciones de roles de desempeño entre jugadoras de primera y segunda línea. Se modeló a partir de ejercicios que involucran el trabajo con el pívot, atendiendo al ataque sobre bloqueo, con acciones de pase y va, cruces para el logro de la creación de espacios que permita la penetración hacia el área de portería.

En la creación de espacios en las situaciones planteadas, las jugadoras a la ofensiva optaron por un juego individual, pues no aprovecharon las opciones de pase y va. Lo 
mismo ocurrió con la casi nula relación de ataque sobre el bloqueo del pívot, al realizarse sin cruce alguno que mostrara variabilidad en el ataque. De este modo se manifestó poca consistencia en el aprendizaje de las acciones ofensivas. El diagnóstico se aplicó sobre la base del ejercicio No. 5, incluido en la metodología con sus variantes. En el cual se realizaron 121 acciones, de las cuales 53 se consideraron positivas, mostrando $43.80 \%$ de efectividad.

Tabla 1. Progresión de acciones de grupo

\begin{tabular}{|c|c|c|c|c|c|c|c|c|}
\hline \multirow{3}{*}{ Grupo } & \multicolumn{6}{|c|}{ Medios tácticos grupales ofensivos } & \multirow{2}{*}{\multicolumn{2}{|c|}{$\begin{array}{c}\text { Resultado } \\
\text { general }\end{array}$}} \\
\hline & \multicolumn{2}{|c|}{ Pase y va } & \multicolumn{2}{|c|}{ Penetraciones } & \multicolumn{2}{|c|}{ Cruces } & & \\
\hline & NP & $P$ & NP & $P$ & $\begin{array}{l}N \\
P\end{array}$ & $P$ & NP & $P$ \\
\hline 1 & & $x$ & $x$ & & $x$ & & & \\
\hline 2 & $x$ & & $x$ & & & $x$ & & \\
\hline 3 & $x$ & & & $x$ & $x$ & & & \\
\hline
\end{tabular}

Fuente: Elaboración propia

Tabla 2. Progresión de acciones de grupo

\begin{tabular}{|c|c|c|c|c|c|c|c|c|}
\hline \multirow{3}{*}{ Grupo } & \multicolumn{6}{|c|}{ Medios tácticos grupales defensivos } & \multirow{2}{*}{\multicolumn{2}{|c|}{$\begin{array}{c}\text { Resultado } \\
\text { general }\end{array}$}} \\
\hline & \multicolumn{2}{|c|}{$\begin{array}{c}\text { Cambio de } \\
\text { oponente }\end{array}$} & \multicolumn{2}{|c|}{ Ayudas } & \multicolumn{2}{|c|}{ Doblaje } & & \\
\hline & NP & $\mathbf{P}$ & NP & $\mathbf{P}$ & NP & $\mathbf{P}$ & NP & $\mathbf{P}$ \\
\hline 1 & $\mathbf{x}$ & & $\mathbf{X}$ & & $\mathbf{x}$ & & & \\
\hline 2 & & $x$ & $\mathbf{x}$ & & & $\mathbf{x}$ & & \\
\hline 3 & X & & & $\mathbf{x}$ & $\mathbf{x}$ & & & \\
\hline
\end{tabular}

Fuente: Elaboración propia 
El resultado obtenido en el trabajo 3vs3 se determinó a partir de valores que oscilaron por debajo del $60 \%$ de efectividad, atendiendo al número de oportunidades aprovechadas en cada una de las situaciones propuestas por el entrenador. Tales resultados instan a realizar precisiones en el proceso de enseñanza aprendizaje del meso ciclo objeto de diagnóstico.

Acciones de ajuste de la preparación, tras los resultados de la primera serie de diagnóstico:

a) Precisión en la relación desplazamiento-pase para el logro del ataque con continuidad de juego

b) Ataque por medio del desdoble entre jugadoras para la creación del espacio

c) Aumento del tiempo de trabajo, atendiendo a las acciones de 3vs3

d) Utilización de variante de ejercicios con ventaja numéricas hasta llegar al 4vs4

2da serie: ubicada en el 2 do meso ciclo básico desarrollador en la etapa de preparación general, del periodo preparatorio, micro ciclo 14.

\section{Resultados del diagnóstico en la 2da serie}

Se percibió mejoría en la consistencia del aprendizaje, a través de la aplicación de los medios tácticos ofensivos desde las variantes con desventajas numéricas hasta el trabajo en igualdad de condiciones (3vs1, 3vs2, 3vs3). Se establecen las relaciones de roles de desempeño entre jugadoras de primera y segunda línea.

En la creación de espacios en las situaciones planteadas, las jugadoras a la ofensiva prestaron mayor atención a la cooperación entre ellas. Tal accionar se evidenció al mostrar sus habilidades para realizar el ataque impar y lograr la penetración para el lanzamiento a portería con una marcada intención de pase. De este modo, se manifiesta la consistencia en el aprendizaje de las acciones ofensivas.

Desde el punto de vista defensivo, las jugadoras que interactúan, mostraron buen control del adversario. Se mantienen las características de la serie anterior, relativas al cambio de oponente, la calidad en el ataque, la ayuda y el doblaje, aunque los dos últimos aspectos, evidenciaron una ligera mejoría.

El resultado obtenido en el trabajo 3vs3 mostró avances con relación a la serie anterior, a partir de los ejercicios que comprenden las relaciones entre ambas líneas de ataque. En este sentido, se destaca la utilización de variantes sobre la base de los 
desdobles para crear y ocupar los espacios. De 138 acciones ofensivas 93 se manifestaron de forma positiva, en representación del $67.3 \%$ de efectividad, según el número de oportunidades en cada una de las situaciones planteadas por el entrenador. Los resultados aún convidan a precisar aspectos esenciales del proceso en el meso ciclo objeto de diagnóstico.

Aun cuando en no pocas ocasiones las acciones de 3vs3 se manifiestan de forma progresiva, persisten dificultades relativas a la integración de las jugadoras con los otros subgrupos colindantes.

Tabla 3. Progresión de acciones de grupo

\begin{tabular}{|c|c|c|c|c|c|c|c|c|}
\hline \multirow{2}{*}{ Grupo } & \multicolumn{9}{|c|}{ Medios tácticos grupales ofensivos } & \multicolumn{2}{c|}{$\begin{array}{c}\text { Resultado } \\
\text { general }\end{array}$} \\
\cline { 2 - 9 } & Pase y va & \multicolumn{2}{c|}{ Penetraciones } & \multicolumn{2}{c|}{ Cruces } & \multicolumn{2}{c|}{} \\
\cline { 2 - 9 } & NP & $P$ & NP & $P$ & NP & $P$ & NP & $P$ \\
\hline 1 & & $x$ & & $x$ & & $x$ & & \\
\hline 2 & & $x$ & & $x$ & & $x$ & & \\
\hline 3 & $x$ & & & $x$ & $x$ & & & \\
\hline
\end{tabular}

Fuente: Elaboración propia

Desde el punto de vista defensivo, las jugadoras que interactúan, mostraron buen control del adversario. Se mantienen las características de la serie anterior, relativas al cambio de oponente, la calidad en el ataque, la ayuda y el doblaje, aunque los dos últimos aspectos, evidenciaron una ligera mejoría.

El resultado obtenido en el trabajo 3vs3 mostró avances con relación a la serie anterior, a partir de los ejercicios que comprenden las relaciones entre ambas líneas de ataque. En este sentido, se destaca la utilización de variantes sobre la base de los desdobles para crear y ocupar los espacios. De 138 acciones ofensivas 93 se manifestaron de forma positiva, en representación del 67.3\% de efectividad, según el 
número de oportunidades en cada una de las situaciones planteadas por el entrenador. Los resultados aún convidan a precisar aspectos esenciales del proceso en el meso ciclo objeto de diagnóstico.

Aun cuando en no pocas ocasiones las acciones de 3vs3 se manifiestan de forma progresiva, persisten dificultades relativas a la integración de las jugadoras con los otros subgrupos colindantes.

Valoración final de los resultados del cuasi experimento y los modos de actuación en el equipo

La aplicación del cuasi experimento permitió constatar las transformaciones operadas no solo en el desempeño táctico del equipo que emerge del aprendizaje. También reflejó la evolución en los modos de actuación profesional de los entrenadores para desarrollar el diagnóstico y potenciar, desde su participación activa y consciente, el rediseño de su accionar conforme a la metodología propuesta.

En lo particular, los entrenadores demostraron la capacidad de retroalimentar de manera sistemática el proceso de preparación deportiva. Connotan la significatividad de la investigación y coinciden en la relevancia de los ejercicios y los registros de medición propuestos, atendiendo a las exigencias del proceso. De igual modo, valoran el desarrollo del cuasi experimento para la implementación del aporte, como vía demostrativa y descriptiva de la realidad en correspondencia con las exigencias del deporte en cuestión.

Las jugadoras, por su parte, evidencian un salto cualitativo en la interpretación de su desempeño tanto en condiciones de entrenamiento como en el juego. Al mismo tiempo, manifiestan independencia cognoscitiva al establecer sus propias estrategias de aprendizaje, a escala individual, grupal y colectiva. De forma general, se alcanza la creatividad adecuada y se solventan barreras personales sobre la base de una interdependencia positiva que, a su vez favorece la resolución de las situaciones tácticas y aporta a la efectividad del equipo.

Las precisiones referidas contribuyen a la concreción de la preparación desde el componente táctico con predominio hacia lo técnico y lo físico. Al mismo tiempo, promueven una valiosa alternativa que contradice el entrenamiento tradicional y connotan la visión contemporánea del entrenamiento deportivo. 


\section{CONCLUSIONES}

1. Los fundamentos teórico-metodológicos del proceso de evaluación del aprendizaje táctico en el balonmano permiten comprender la necesidad de incursionar en su función de diagnóstico

2. El análisis del estado actual del aprendizaje táctico en el equipo de balonmano categoría juvenil del sexo femenino en Honduras posibilitó la identificación de las carencias y potencialidades de la práctica, así como las limitaciones normativas inherentes a la problemática

3. La metodología para el diagnóstico del aprendizaje táctico en el equipo de balonmano categoría juvenil del sexo femenino de la propuesta, satisface los requerimientos al estructurarse en tres fases; ofrece ejercicios, indicaciones y registros de medición que contextualizan los indicadores didácticos de consistencia y progresión al diagnóstico del aprendizaje táctico en el balonmano en condiciones de entrenamiento y juego

4. Los resultados que emergen de la aplicación de un cuasi experimento y del método criterio de especialista permiten valorar las transformaciones operadas en el desempeño de entrenadores y del equipo de balonmano, además se infiere en la aceptación y factibilidad de la metodología como alternativa para el diagnóstico del aprendizaje táctico.

\section{REFERENCIAS BIBLIOGRÁFICAS}

Addine, F. (1998). Didáctica y optimización del proceso de enseñanza-aprendizaje. IPLAC, La Habana

Addine, F. y González, M. (s.f). Principios para la dirección del proceso pedagógico. Facultad de Ciencias de la Educación, ISP "Enrique José Varona"

Alcarde, A. (1997). Análisis y Evaluación en Balonmano. Recuperado de: http://www.efdeportes.com

Almaguer, R. (2000). Las situaciones simplificadas de juego: condición metodológica para iniciar al balonmano. Tesis de maestría. ISCF, Santiago de Cuba

Almaguer, R. (2003). Evaluación de la táctica en el balonmano. La Habana: Científico-Técnica

Almaguer, R. (2004). "Estudio comparativo entre dos metodología de enseñanza: Una aplicación en la investigación del balonmano". Tesis doctoral. ISCF, La Habana

Álvarez, A. (2003). Estrategia, táctica y técnica: definiciones características y ejemplo de los controvertidos términos. Recuperado de http://www efdeporte.com / efdo / b-hand.htm 
Álvarez, C. (1995). Metodología de la investigación científica. Santiago de Cuba: Universidad de Oriente

Antón, J.L. (1992). Balonmano. Fundamentos y etapas de aprendizaje. Gymnos, SA. Madrid: Deportiva

Antón, J. L. (1998). Balonmano, táctica grupal ofensiva. Conceptos, estructura y metodología. Gymnos, SA. España: Deportiva Madrid

Antón, J. (2010). Uso del portero falso en inferioridad numérica atacante: ¿nueva aportación táctico-estratégica? Recuperado de: http//:www.e-balonmano.com

Antón, J. (2014). El aprendizaje del pase en balonmano: valoración de sus posibilidades de tratamiento según el nivel de complejidad ejecutiva. Revista Apunts. INEFC

Arias, J. L. (2007). Estructuración del sistema de ataque 2-4 en balonmano. Recuperado de: http/l:www.e-balonmano.com

Bayer, C. (1986). Técnica del balonmano. La formación del jugador. Barcelona: Hispano Europea

Brueckner, L. (1975). Diagnóstico y tratamiento de las dificultades en el aprendizaje. La Habana: Edición revolucionaria

Contreras, O. (1998). Didáctica de la educación física. Un enfoque constructivista. INDE:España

Cordovés, R. (2011). Indicadores para el control de rendimiento técnico táctico ofensivo en el balonmano. Disponible en: mlaoc@scu.uccfd.cu

Cordovés, R., Infante, M. y Mesa, L. (2015). El control del aprendizaje en la actividad táctica y sus particularidades psicológicas. No. 2140. Recuperado de: http://www.revista.iplac.rimed.cu

Cordovés, R., Mesa, L. y Sánchez, L. (2014). Estrategia didáctica de control del aprendizaje en la actividad táctica. Recuperado de: http://www.revista.iplac.rimed.cu

Cordovés, R. (2015). La evaluación del aprendizaje en la actividad táctica en el equipo de balonmano. Tesis doctoral no publicada. Universidad de Oriente, Facultad de Cultura Física, Santiago de Cuba

Czerwinski, J. (1993). El balonmano: técnica y entrenamiento. Barcelona: Paidotribo

Cruz, J. A. y Col. (1996). Programa de preparación del deportista: Balonmano. La Habana

Dick, F. (1993). La evaluación en el deporte. Barcelona: Paidotribo

Espar, X. y Gerona, T. (2004). Capacidades cognoscitivas y tácticas en los deportes de equipo. [Documento en línea]. Disponible en: mlaoc@scu.uccfd.cu

Forcades, L. (1998). Guía metodológica de balonmano. ISCF, La Habana 